\title{
Viscoelastic behavior of an ordering latex suspension in a steady shear flow
}

\author{
B. van der Vorst, D. van den Ende, ${ }^{*}$ N. A. Tekin, and J. Mellema \\ J.M. Burgerscentrum, Rheology Group, Department of Applied Physics, University of Twente, \\ P.O. Box 217, 7500 AE Enschede, The Netherlands
}

(Received 16 September 1997)

\begin{abstract}
The linear viscoelastic behavior of an ordering polystyrene latex during steady shear flow has been investigated. In this study a home-made instrument has been used that superimposes a small-amplitude oscillatory shear orthogonal onto the steady shear flow. The measurements can be interpreted as due to the coexistence of two phases under flow. For increasing shear rate the ordered (or solidlike) phase melts away until the suspension becomes completely disordered (or fluidlike). A model is presented in which the fluid is conceived as a viscoelastic matrix containing spherical viscoelastic domains. The model predicts a similar linear viscoelastic behavior of sheared lattices as found experimentally. The resulting parameters give information about the microstructure of the dispersion under shear flow. [S1063-651X(98)15002-X]
\end{abstract}

PACS number(s): 83.50.Fc

\section{INTRODUCTION}

In recent years a lot of work has been done both experimentally and theoretically, to investigate and understand the linear viscoelastic behavior of ordering latices at rest (for a compilation of data see [1]). The shear viscosity of these latices has been studied experimentally, for instance, by Chen and Zukoski [2], Buscall [3], and Quemada [4]. Theoretical models have been given by Buscall [3] and Quemada [4]. To describe the measured viscosity as a function of shear rate and volume fraction more quantitatively a microrheological model has recently been proposed by Van der Vorst et al. [5]. This model was based on the hypothesis that in a steady shear flow the latex dispersion consists of ordered domains, space filling at rest, which melt away shear induced, in favor of the disordered fluid phase as observed by Imhof et al. [6]. The linear viscoelastic behavior of these ordering latices during steady shear flow has been investigated in order to corroborate or falsify this picture.

Linear viscoelastic measurements on an ordered latex at rest give information about the microstructure of the suspension at rest. The steady shear viscosity of the ordering latex will be determined by the microstructure of the suspension in shear flow. This viscosity gives some information about the shear rate dependence of the microstructure. A transition of the shear viscosity as a function of the shear rate indicates a change of the microstructure in the fluid. However, specific information about the morphology of the microstructure is difficult to obtain from such a flow curve and is model dependent. In order to probe the microstructure of the latex in shear flow, dynamic measurements were performed on a sheared latex. A home-made instrument was used that superimposes a small-amplitude harmonic shear orthogonal to a steady shear flow [7]. Although the experimental method has been used before [8], this is, as far as we know, the first application in dispersion rheology. The experiments are described in Sec. III. In Sec. II the theoretical model for the dynamic viscosity for an emulsion of viscoelastic droplets in

\footnotetext{
*Author to whom correspondence should be addressed.
}

a non-Newtonian fluid is described. Finally, in Sec. IV the experimental and theoretical results are discussed.

\section{THEORY}

The linear viscoelastic behavior of an ordering latex dispersion during steady shear flow is described with a model originally developed for a nondilute emulsion of deformable spheres $[9,10]$. The latex suspension is modeled as a dispersion of spherical crystalline aggregates dispersed in a linear viscoelastic fluid. The aggregates contain latex particles ordered in a crystal array and the viscoelastic fluid consists of disordered latex (see Fig. 1). The particle densities in both phases are kept equal. The linear viscoelasticity of the crystalline aggregates is described with a Maxwell fluid:

$$
G_{\mathrm{cr}}^{*}=G_{\infty}^{\mathrm{cr}} \frac{\left(\omega \tau_{\mathrm{cr}}\right)^{2}+i \omega \tau_{\mathrm{cr}}}{1+\left(\omega \tau_{\mathrm{cr}}\right)^{2}}+i \omega \eta_{\infty} .
$$

$G_{\mathrm{cr}}^{*}$ has been taken equal to the shear modulus of the dispersion at rest. The disordered liquid phase is also modeled as a Maxwell fluid with complex viscosity:

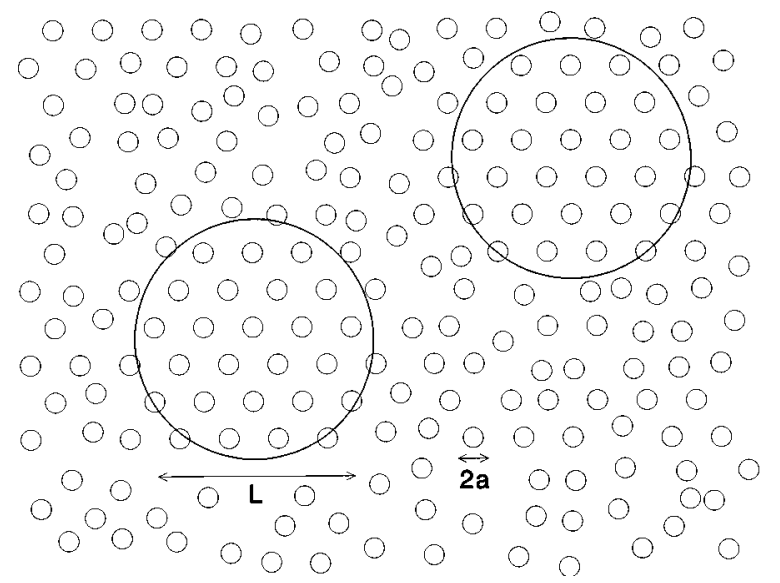

FIG. 1. The suspension is modeled as an emulsion of spherical crystallites (droplets) of size $L$ in a disordered (fluidlike) phase. 
TABLE I. The coefficients $\alpha_{n}$ expressed in $E=\eta_{\mathrm{cr}}^{*} / \eta_{\mathrm{fl}}^{*}$.

\begin{tabular}{cc}
\hline \hline$n$ & $\alpha_{n}(E)$ \\
\hline 0 & $76(1-E)(1-E)$ \\
3 & $25(1-E)(18+19 E)$ \\
5 & $42(E-1)(16+19 E)$ \\
7 & $25(1-E)(16+19 E)$ \\
10 & $2(3+2 E)(16+19 E)$ \\
\hline \hline
\end{tabular}

$$
\eta_{\mathrm{fl}}^{*}=\left(\eta_{\mathrm{fl}}-\eta_{\infty}\right) \frac{1-i \omega \tau_{\mathrm{fl}}}{1+\left(\omega \tau_{\mathrm{fl}}\right)^{2}}+\eta_{\infty}
$$

Here $\tau_{\mathrm{fl}}=\left(\eta_{\mathrm{fl}}-\eta_{\infty}\right) / G_{\infty}^{\mathrm{fl}}$ while $G_{\infty}^{\mathrm{cr}}$ and $G_{\infty}^{\mathrm{fl}}$ are the high frequency shear moduli at rest for the ordered and disordered phase, respectively; $\eta_{\infty}$ is the high frequency viscosity at rest, which is chosen equal for the ordered and disordered phase in the suspension; $\eta_{\mathrm{fl}}=\eta_{\mathrm{fl}}(\dot{\gamma})$ is the viscosity of the
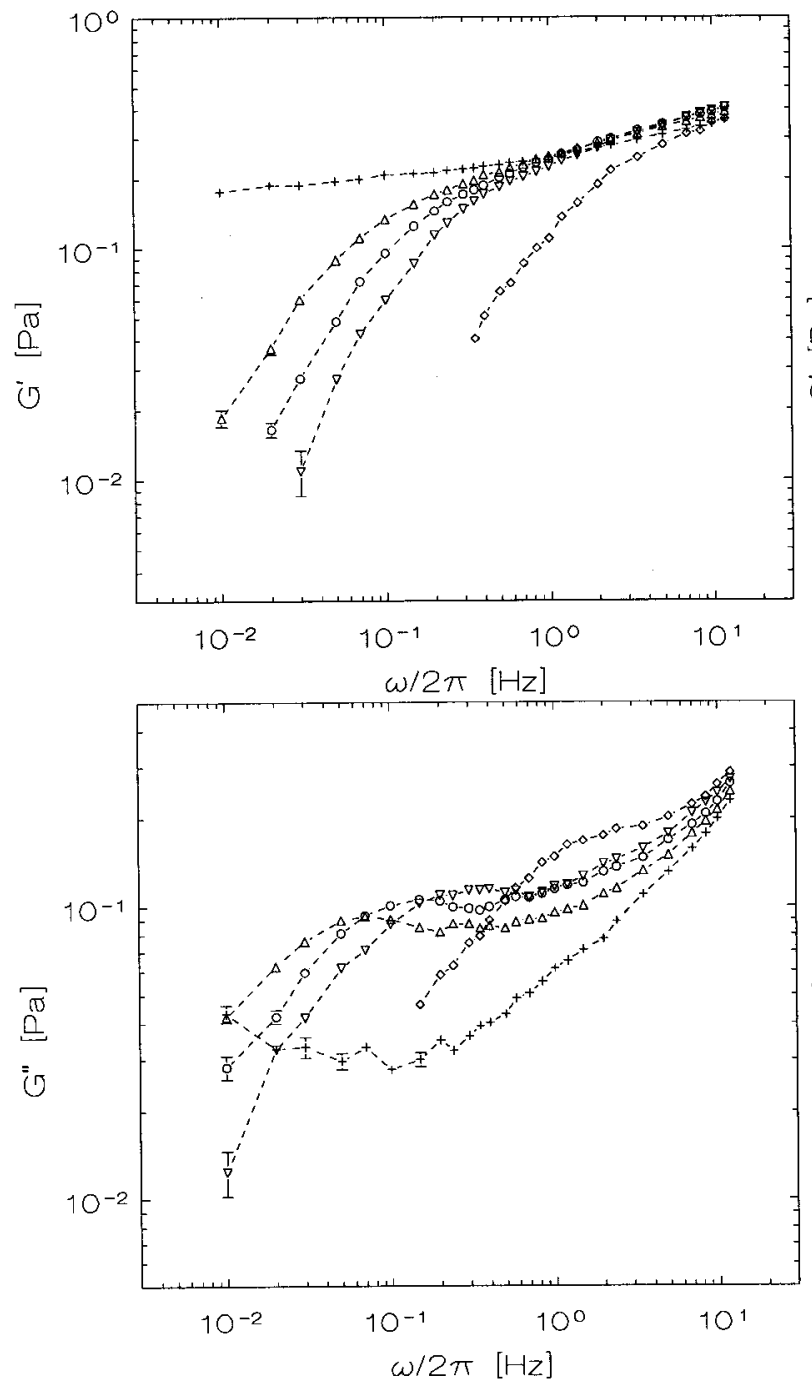

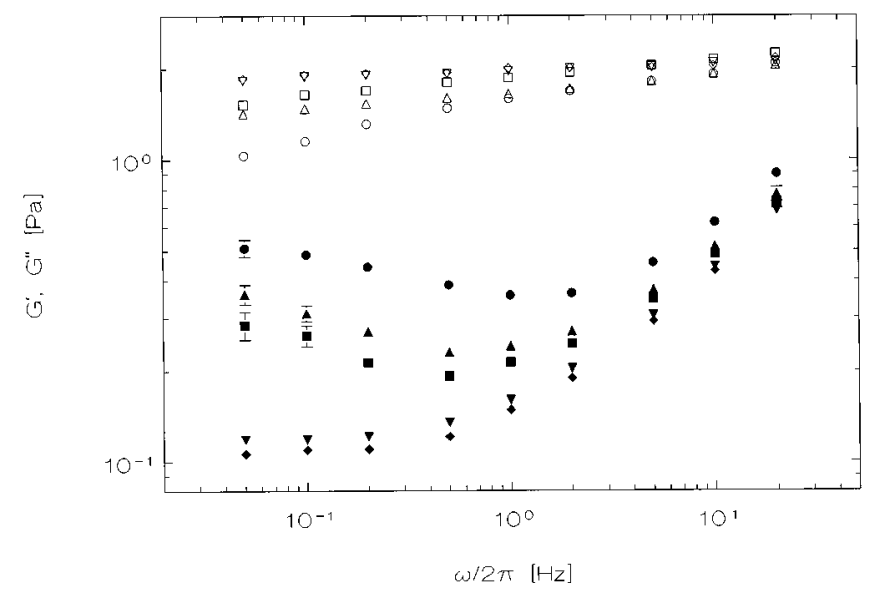

FIG. 2. The storage modulus (open symbols) and the loss modulus (solid symbols) as a function of the frequency at zero shear rate for several preshear times. The duration of the applied preshear of $\dot{\gamma}=1.25 \mathrm{~s}^{-1}$ was $0 \min (\bigcirc), 4 \min (\triangle), 6 \min (\square), 10 \min (\nabla)$, $36 \min (\diamond), \phi=0.20$.
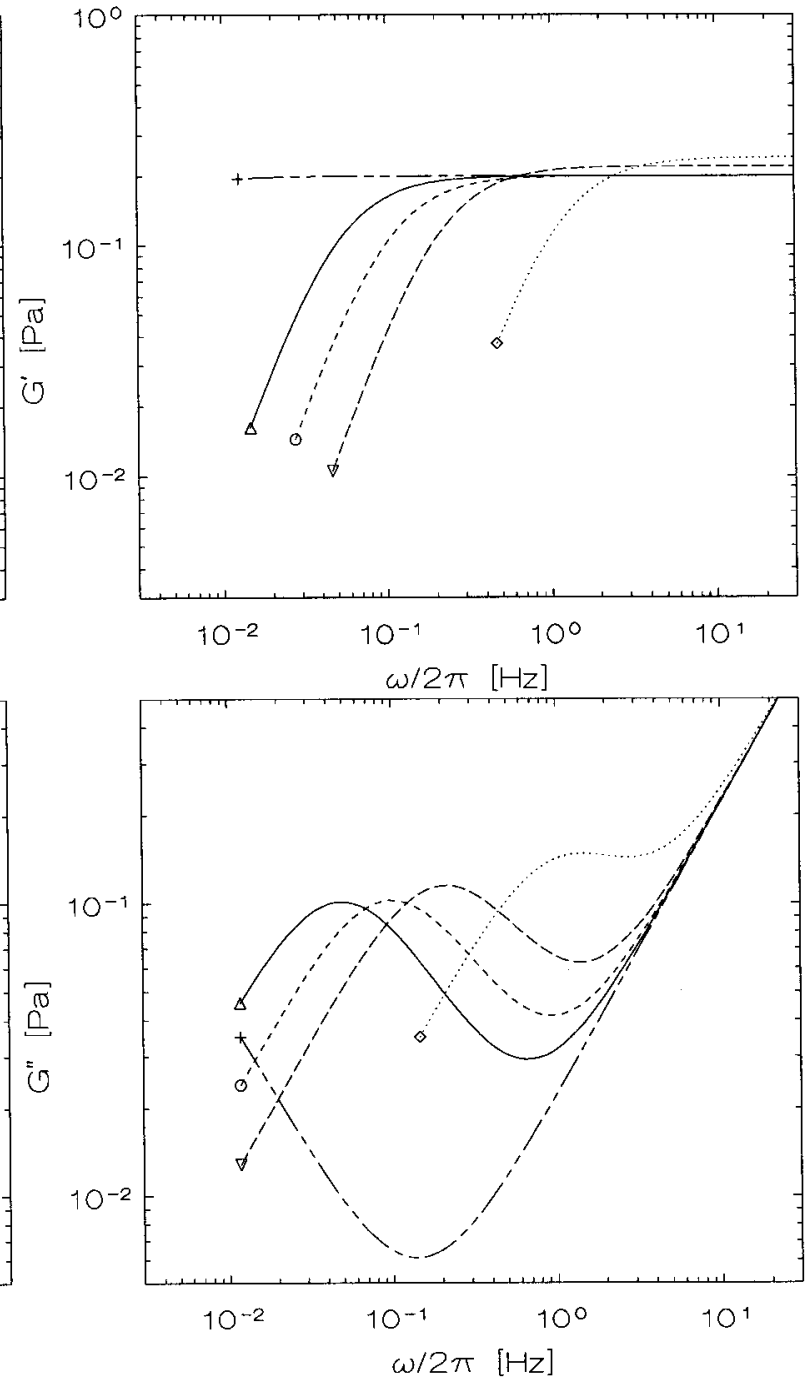

FIG. 3. The storage modulus (upper two figures) and the loss modulus (lower two figures) as a function of the frequency for a volume fraction $\phi=0.10$. The two figures on the left-hand side show the experimental results and the figures on the right-hand side show the curves fitted to these experiments. The applied shear rates are $0 \mathrm{~s}^{-1}(+), 0.0125 \mathrm{~s}^{-1}(\triangle), 0.025 \mathrm{~s}^{-1}(\diamond), 0.05 \mathrm{~s}^{-1}(\nabla), 0.5 \mathrm{~s}^{-1}(\diamond)$. 

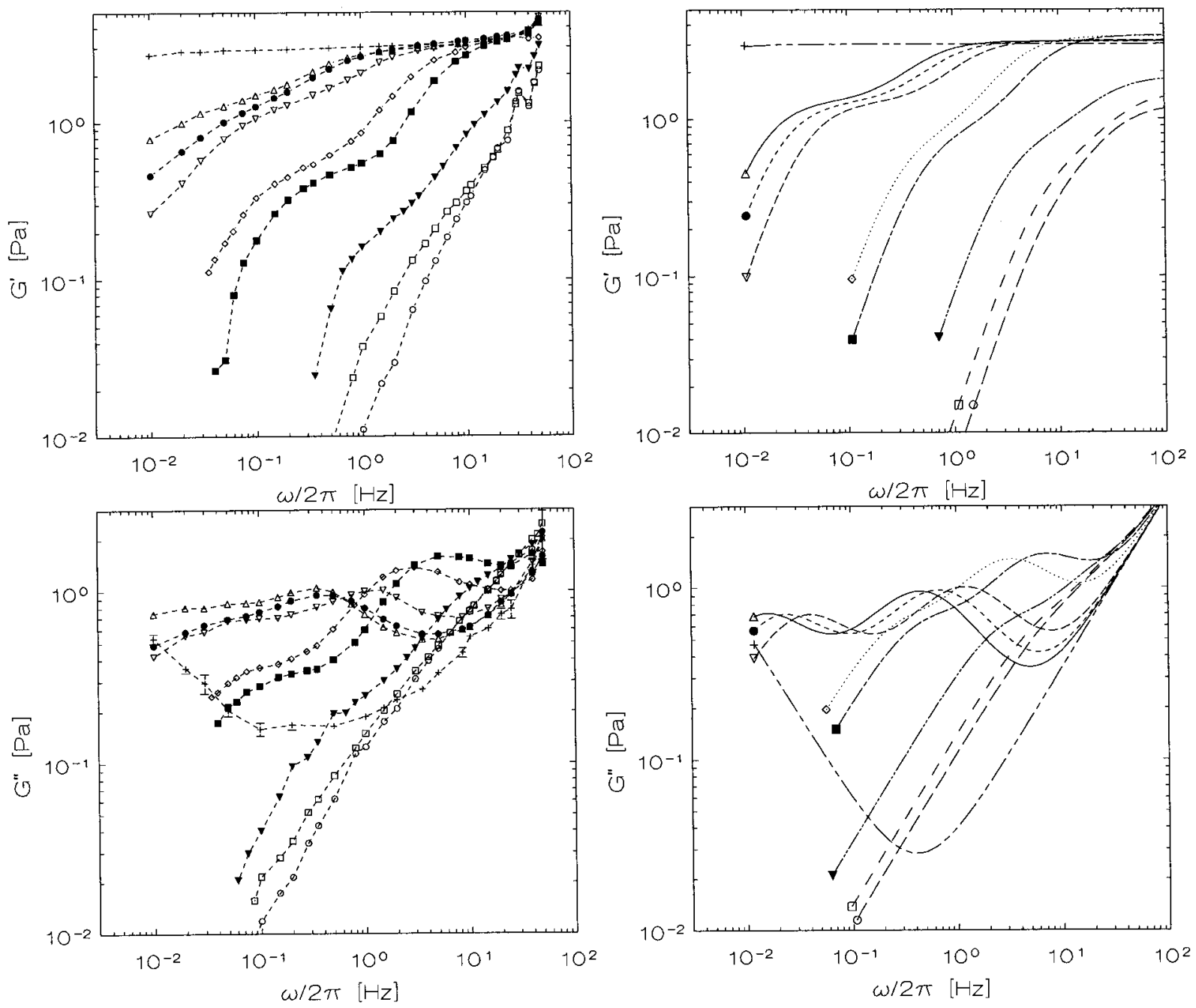

FIG. 4. The storage modulus (upper two figures) and the loss modulus (lower two figures) as a function of the frequency for a volume fraction $\phi=0.20$. The two figures on the left-hand side show the experimental results and the figures on the right-hand side show the curves fitted to these experiments. The applied shear rates are $0 \mathrm{~s}^{-1}(+), 0.0125 \mathrm{~s}^{-1}(\triangle), 0.025 \mathrm{~s}^{-1}(\mathbf{O}), 0.05 \mathrm{~s}^{-1}(\nabla), 0.5 \mathrm{~s}^{-1}(\diamond), 1.25 \mathrm{~s}^{-1}$ $(\square), 5 \mathrm{~s}^{-1}(\boldsymbol{\nabla}), 10 \mathrm{~s}^{-1}(\square), 25 \mathrm{~s}^{-1}(\bigcirc)$.

disordered fluid phase, which depends on the steady shear rate $\dot{\gamma}$. Graebling and Muller [11] describe an emulsion model of two viscoelastic liquids. This model is accurate up to order $\phi$ where $\phi$ is the volume fraction of the dispersed phase. The volume fraction of crystalline aggregates, $\phi_{\mathrm{cr}}$, however, will be rather high at low shear rates, so we are interested in a model for $G^{*}$ that is also applicable at high $\phi_{\mathrm{cr}}$. Therefore an effective medium cell model for the linear viscoelasticity of emulsions proposed by Oosterbroek and Mellema [10] has been modified. In this model the spherical aggregate with radius $r_{a}=L / 2$ is at the center of a cell with radius $r_{c}$. Within this cell the fluid surrounding the aggregate has a viscosity $\eta_{\mathrm{fl}}^{*}$ and the aggregate $\eta_{\mathrm{cr}}^{*}=G_{\mathrm{cr}}^{*} / i \omega$. The oscillatory flow problem is solved for small deformations when the aggregates stay almost spherical. Therefore the rotational part of the flow may be neglected and only the pure straining contribution is considered. At the outer boundary of the cell the flow field is supposed to match the macroscopic flow field. At the boundary of the aggregate the deformation and stresses inside the aggregate match those inside the fluid, because there is no surface tension for the aggregate assumed. From the calculated flow and stress field (see Oosterbroek and Mellema [10] and the Appendix for details) an expression for the complex shear modulus of the dispersion is obtained:

$$
G^{*}=i \omega \eta_{\mathrm{fl}}^{*}\left(1-\frac{f^{3}\left(\frac{2}{5} \alpha_{7} R^{7}+\frac{5}{2} \alpha_{0}\right)}{\alpha_{10} R^{10}+\alpha_{7} R^{7}+\alpha_{5} R^{5}+\alpha_{3} R^{3}+\alpha_{0}}\right),
$$

where $R=r_{c} / r_{a}$. The expressions for $\alpha_{n}(E)$ are listed in Table I where $E=\eta_{\mathrm{cr}}^{*} / \eta_{\mathrm{ff}}^{*}$. The distance $r_{c}$ can be regarded as the effective hydrodynamic interaction radius. The relation between $r_{c}$ and $\phi_{\mathrm{cr}}$ was taken equal to that proposed by Thomas [12]: $r_{c}=f r_{a} \phi_{\mathrm{cr}}^{-1 / 3}$. He found that there was good agreement with experiments for $f=1.111$ when he compared Simha's theory [13] for the suspension viscosity [which is equal to $\left.\lim _{\omega \rightarrow 0}\left(G^{\prime \prime}(\omega) / \omega\right)\right]$ with high-shear steady-state viscosity data on suspensions of rigid spheres. For low vol- 

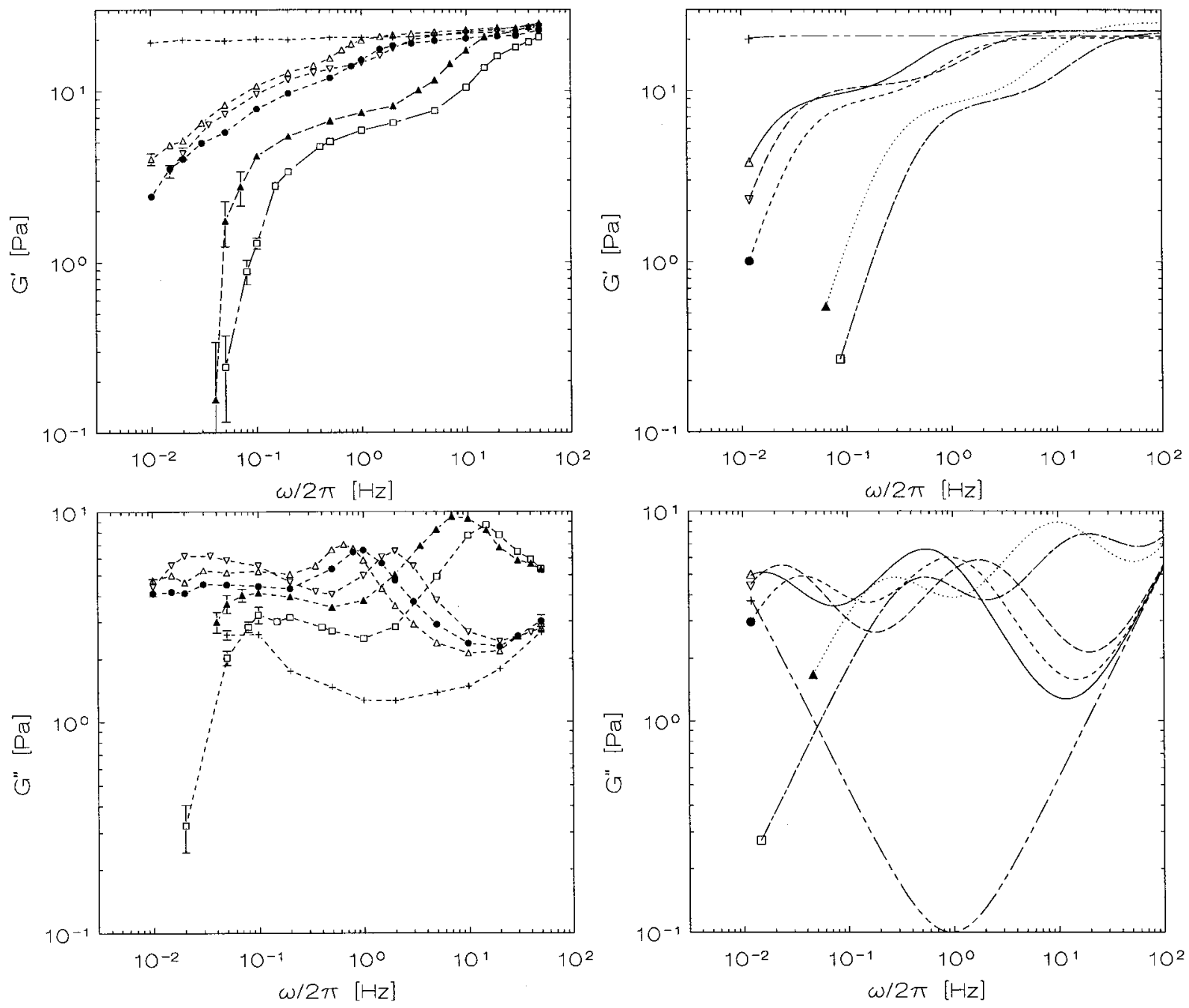

FIG. 5. The storage modulus (upper two figures) and the loss modulus (lower two figures) as a function of the frequency for a volume fraction $\phi=0.30$. The two figures on the left-hand side show the experimental results and the figures on the right-hand side show the curves fitted to these experiments. The applied shear rates are $0 \mathrm{~s}^{-1}(+), 0.0125 \mathrm{~s}^{-1}(\triangle), 0.025 \mathrm{~s}^{-1}(\boldsymbol{\bullet}), 0.05 \mathrm{~s}^{-1}(\nabla), 0.5 \mathrm{~s}^{-1}(\boldsymbol{\Delta}), 1.25 \mathrm{~s}^{-1}$ $(\square)$

ume fractions of aggregates this expression for $G^{*}$ is equivalent to the result obtained by Graebling and Muller [11]. The dynamic modulus $G^{*}$ that results from this model shows two separate transitions. The transition at low frequencies pertains to the relaxation process in which a deformed aggregate relaxes to its equilibrium state. The strength of this transition decreases with decreasing volume fraction of the ordered phase (droplets) and also its typical time scale decreases since it is proportional to $\eta / G_{\infty}^{\mathrm{cr}}$ where the viscosity of the dispersion, $\eta$, decreases with decreasing $\phi_{\mathrm{cr}}$, while $G_{\infty}^{\mathrm{cr}}$ is a constant. The second transition occurs at a higher frequency and stems from the fluid phase, which has been modeled with a Maxwell model according to Eq. (2). The strength of this transition increases with decreasing droplet volume fraction.

\section{EXPERIMENTS}

\section{A. The model fluid}

In the present study, we use a monodisperse polystyrene latex that has been prepared following the method described by Goodwin et al. [14]. A comprehensive survey of the preparation of latices is given by Hearn et al. [15]. The purification and characterization of the samples have been described in detail in a previous article [1]. The latex dispersions used for our experiments are characterized by the particle radius $a$, the surface charge density on the particles $\sigma$, the excess electrolyte concentration $n_{b}$, and the volume fraction $\phi$. The particle radius $a=195 \mathrm{~nm}$ was obtained from electron microscopy and the surface charge density $\sigma=$ $-4.6 \mu \mathrm{C} / \mathrm{cm}^{2}$ from conductometric titration. The electrolyte concentration of the latex has been reduced with an ionexchange resin as much as possible. The resulting electrolyte concentration is about $n_{b}=10 \mu M$ [1]. Samples with volume fractions $\phi=0.10,0.20,0.30$, and 0.35 were used.

\section{B. Apparatus}

To study the complex or dynamic shear viscosity of ordered latices during steady shear flow, a home-made instrument was used, that applies a small amplitude harmonic shear orthogonal on a steady shear flow. This instrument has 

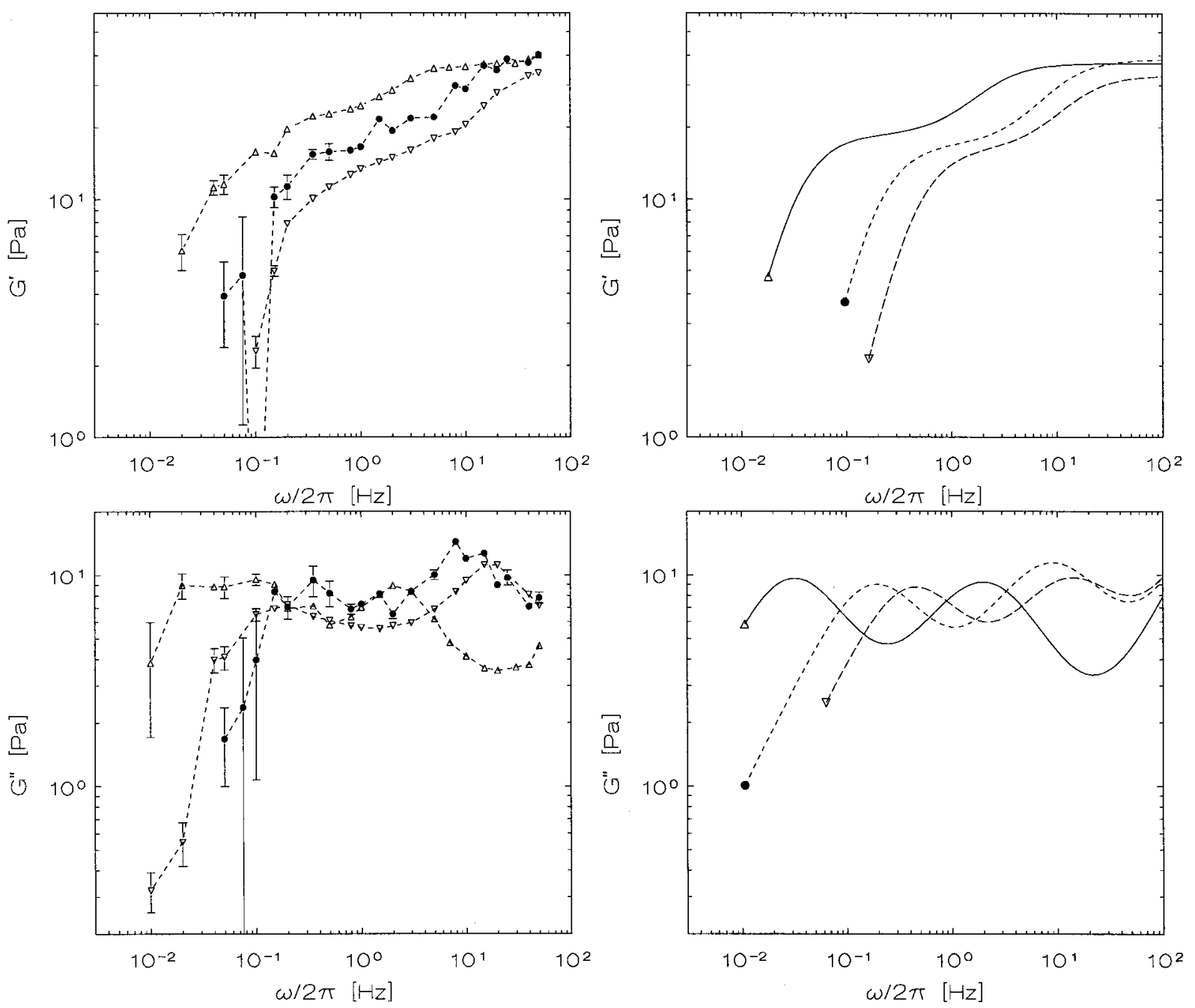

FIG. 6. The storage modulus (upper two figures) and the loss modulus (lower two figures) as a function of the frequency for a volume fraction $\phi=0.35$. The two figures on the left-hand side show the experimental results and the figures on the right-hand side show the curves fitted to these experiments. The applied shear rates are $0.05 \mathrm{~s}^{-1}(\triangle), 0.5 \mathrm{~s}^{-1}(\bullet), 1.25 \mathrm{~s}^{-1}(\nabla)$.

been described in detail by Zeegers et al. [7]. It consists of a thin walled cylinder that oscillates in an axial direction in an annular sample holder. The cylinder is suspended from a spring, which in turn is attached to a linearly oscillating exciter. The annular beaker can be rotated, providing the steady shear flow. From the measured phase difference $\varphi$ and amplitude ratio $R$ of the oscillatory displacements of exciter and cylinder the fluid impedance $Z^{*}$ is obtained and from $Z^{*}$ the complex shear modulus $G^{*}$ can be calculated. The instrument operates in the frequency range from $10^{-3}$ to $50 \mathrm{~Hz}$ and the steady shear rate $\dot{\gamma}$ ranges from 0.01 to $100 \mathrm{~s}^{-1}$. The oscillatory shear amplitude of the instrument can be varied between 0.003 and 3, so linearity of the measurements can be checked.

\section{Measuring procedure}

Since we are interested in the linear viscoelastic behavior of the latices, the linear regime for given experimental conditions was determined before every measurement by measuring the dependency of the complex modulus on the am- plitude of the shear strain. From this investigation we obtained a critical shear strain $\gamma_{c}=0.02$ below which the storage modulus $G^{\prime}$ and the loss modulus $G^{\prime \prime}$ do not depend on the shear strain $\gamma$. The critical shear was almost independent of applied frequency, shear rate, and volume fraction. These observations are in line with those of Goodwin and Hughes [16]. Also the influence of different shear histories of the latices due to the rather uncontrollable filling process of the instrument should be investigated and if possible minimized by applying a well-defined shear history. To that end the samples were presheared at $1.25 \mathrm{~s}^{-1}$. The behavior of the complex modulus at a certain shear rate was measured after different preshear times. The complex modulus measured at zero shear rate depends most significantly on the shear history. This case is shown in Fig. 2. From this figure it follows that the behavior of the storage and the loss modulus becomes independent of the preshear time above $10 \mathrm{~min}$ preshearing. Moreover, the storage modulus is flattened by the preshear and the minimum of the loss modulus decreases and shifts to lower frequencies due to the preshear. This indicates that preshearing results in an enhanced (long range) ordering in the sample. We also investigated the time it takes 
TABLE II. The model parameters as obtained from this and previous work [5]; the columns from [5] are indicated with (@); $\tau_{\mathrm{cr}}=80 \mathrm{~s}$ for all values of $\phi$.

\begin{tabular}{|c|c|c|c|c|c|c|c|c|c|c|c|}
\hline$\phi$ & $\begin{array}{c}G_{\infty}^{\mathrm{cr}} \\
{[\mathrm{Pa}]}\end{array}$ & $\begin{array}{c}\eta_{\infty} \\
{[\mathrm{Pa} \mathrm{s}]}\end{array}$ & $\begin{array}{c}\gamma \\
{\left[\mathrm{s}^{-1}\right]}\end{array}$ & $R_{\mathrm{cr}} / a$ & $\phi_{\mathrm{cr}}$ & & $\begin{array}{c}\eta_{\mathrm{fl}} \\
{[\mathrm{Pa} \mathrm{s}]}\end{array}$ & & $\begin{array}{c}\tau_{\mathrm{fl}} \\
{[\mathrm{s}]}\end{array}$ & $\begin{array}{c}\eta_{o} \\
{[\mathrm{~Pa} \mathrm{~s}]}\end{array}$ & \\
\hline & & & & (@) & & (@) & & (@) & & & (@) \\
\hline \multirow[t]{4}{*}{0.1} & 0.2 & 0.003 & 0.0125 & 23 & $?$ & 0.32 & 0.65 & 0.19 & 3.24 & 0.65 & 0.55 \\
\hline & & & 0.025 & 22 & $?$ & 0.31 & 0.33 & 0.10 & 1.64 & 0.33 & 0.28 \\
\hline & & & 0.05 & 20 & $?$ & 0.30 & 0.17 & 0.054 & 0.759 & 0.17 & 0.14 \\
\hline & & & 0.5 & 12 & $?$ & 0.17 & 0.038 & 0.012 & 0.146 & 0.038 & 0.018 \\
\hline \multirow[t]{8}{*}{0.2} & 3.0 & 0.006 & 0.0125 & 46 & 0.45 & 0.48 & 1.05 & 1.69 & 0.326 & 15 & 12.5 \\
\hline & & & 0.025 & 45 & 0.44 & 0.48 & 0.71 & 0.86 & 0.220 & 10 & 6.25 \\
\hline & & & 0.05 & 44 & 0.44 & 0.48 & 0.42 & 0.44 & 0.125 & 5.8 & 3.14 \\
\hline & & & 0.5 & 37 & 0.24 & 0.45 & 0.18 & 0.059 & 0.0497 & 0.56 & 0.35 \\
\hline & & & 1.25 & 30 & 0.26 & 0.42 & 0.097 & 0.033 & 0.0253 & 0.35 & 0.16 \\
\hline & & & 5 & 17 & 0.25 & 0.31 & 0.016 & 0.018 & 0.0067 & 0.053 & 0.050 \\
\hline & & & 10 & 12 & 0.17 & 0.23 & 0.023 & 0.015 & 0.0142 & 0.023 & 0.030 \\
\hline & & & 25 & 8 & 0.13 & 0.09 & 0.011 & 0.012 & 0.0050 & 0.018 & 0.015 \\
\hline \multirow[t]{5}{*}{0.3} & 21 & 0.008 & 0.0125 & 73 & 0.48 & 0.55 & 6.2 & 4.81 & 0.258 & 110 & 65 \\
\hline & & & 0.025 & 73 & 0.45 & 0.55 & 3.1 & 2.43 & 0.155 & 45 & 33 \\
\hline & & & 0.05 & 70 & 0.56 & 0.54 & 2.0 & 1.23 & 0.0797 & 77 & 16 \\
\hline & & & 0.5 & 61 & 0.42 & 0.53 & 0.47 & 0.16 & 0.0159 & 5.9 & 1.8 \\
\hline & & & 1.25 & 50 & 0.43 & 0.50 & 0.22 & 0.087 & 0.0092 & 3.0 & 0.8 \\
\hline \multirow[t]{3}{*}{0.35} & 37 & 0.01 & 0.05 & 105 & 0.55 & 0.58 & 2.6 & 1.25 & 0.0700 & 98 & 25.7 \\
\hline & & & 0.5 & 79 & 0.49 & 0.55 & 0.68 & 0.19 & 0.0168 & 15 & 2.93 \\
\hline & & & 1.25 & 63 & 0.47 & 0.53 & 0.35 & 0.11 & 0.0113 & 6.4 & 1.34 \\
\hline
\end{tabular}

before the complex modulus reaches its equilibrium value after a change in the shear rate. It was found that in the worst case, steady state was reached after 5 min of shearing.

Taking into account these considerations, the following measuring procedure has been set up. After filling the instrument with $10 \mathrm{ml}$ latex, the material is presheared at a shear rate of $1.25 \mathrm{~s} \mathrm{~s}^{-1}$ for $20 \mathrm{~min}$. Subsequently, shearing is stopped and the complex modulus $G^{*}$ is measured as a function of the frequency at zero shear rate. Next $G^{*}$ is measured for increasing steady shear rates after applying this shear rate for $5 \mathrm{~min}$. In order to obtain information on the reproducibility, the experiment is repeated after filling the apparatus again with a new sample.

\section{Results and analysis}

The experimental results for $\phi=0.10,0.20,0.30$, and 0.35 are presented in the left panels of Figs. 3 through 6 , respectively. For all volume fractions, except $\phi=0.10$, the storage and loss moduli measured at low shear rates indicate the existence of at least two transitions. The characteristic frequency of both transitions increases with increasing shear rate. The strength of the low-frequency transition decreases with increasing shear rate. Since the high frequency modulus does not depend strongly on the shear rate, the decrease of the strength of the low frequency transition is more or less compensated by an increasing strength of the high frequency transition so the added strength of the two transitions remains almost constant. In addition for $\phi=0.10$ and 0.20 , the loss modulus measured for different shear rates at high frequencies tends to coincide on a single curve with a constant high-frequency viscosity $\left(G^{\prime \prime}=\eta_{\infty} \omega\right)$.

The measurements are compared with the model described in Sec. II. This model contains six independent parameters: the high-frequency modulus $G_{\infty}^{\mathrm{cr}}$ of the crystallites, $\eta_{\mathrm{fl}}$ of the fluid phase, and the characteristic time scales $\tau_{\mathrm{cr}}$ and $\tau_{\mathrm{fl}}$ of both phases. The fifth parameter is the high frequency viscosity $\eta_{\infty}$, which is taken equal for both the aggregates and the fluid phase. The last parameter in this model is the volume fraction of aggregates $\phi_{\mathrm{cr}}$. The viscoelastic properties of a single crystallite in shear flow are assumed to be identical with that of the dispersion at zero shear rate. So the values for $G_{\infty}^{\mathrm{cr}}, \tau_{\mathrm{cr}}$, and $\eta_{\infty}$ have been determined, for all four volume fractions independently, by comparing the measurements at zero shear rate with the Maxwell model described by Eq. (1). The values found this way for these parameters are used in the analysis of the measurements at nonzero shear rate. Thus, the only remaining parameters are $\eta_{\mathrm{fl}}, \tau_{\mathrm{fl}}$, and $\phi_{\mathrm{cr}}$. These have been determined, for each 

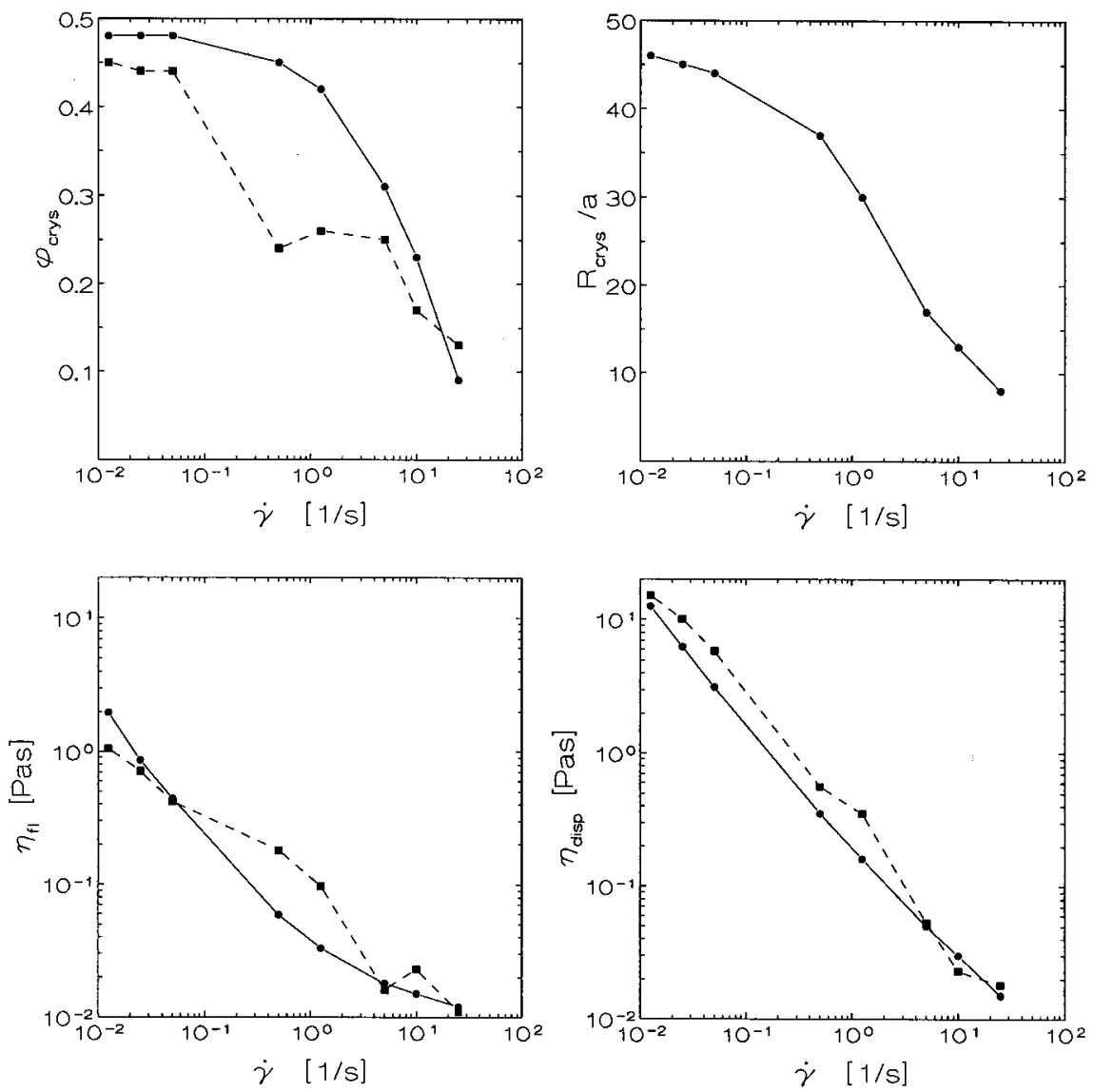

FIG. 7. The volume fraction of ordered domains (upper left), the mean radius of the ordered domains (upper right), the viscosity of the disordered fluid (lower left) and the viscosity of the suspension (lower right figure) as a function of the steady rate of shear for a volume fraction $\phi=0.20$. The solid lines represent the results obtained from the flow curve modeling and the dashed lines the results from the viscoelastic modeling.

shear rate independently, by comparing the model to the experimental results. The obtained values for the parameters are listed in Table II together with $\eta_{0}=\lim _{\omega \rightarrow 0}\left[G^{*}(\omega, \dot{\gamma}) / \omega\right]$. The corresponding curves are shown in the right panels of Fig. 3 through 6. For $\phi=0.10$, where just one transition is visible, no significant contribution from the ordered domains could be obtained so for this value of $\phi$ no values for $\phi_{\mathrm{cr}}$ are given in Table II.

\section{DISCUSSION AND CONCLUSIONS}

From the two distinct transitions observed in the dynamic measurements shown in Figs. 4 through 6 we conclude that there are two sets of relaxation processes acting in the fluid. For a fixed volume fraction the total strength of the two transitions is more or less constant $\left(G_{\infty} \approx\right.$ const $)$ and the strength of the low frequency transition decreases with increasing shear rate. Above observations are consistent with our hypothesis based on the experiments of Imhof et al. [6], that in a steady shear flow the latex dispersion consists of ordered domains, space filling at rest, which melt away in favor of the disordered fluid phase. The model based on this hypothesis describes the experiments quite well. From Table II one observes that, in the frame of this model, not only the viscosity of the dispersion $\left(\eta_{0}\right)$ but also the viscosity of the disordered phase $\left(\eta_{\mathrm{ft}}\right)$ is strongly shear thinning. Moreover the volume fraction of ordered domains, $\phi_{\mathrm{cr}}$, indeed shows a tendency to decrease with increasing $\dot{\gamma}$, which is most clearly for $\phi=0.2$.

The measurements indicate that the complex modulus consists of two sets of relaxation times instead of the two distinct times present in our model. This can be concluded, for instance, from the fact that the local minimum in the loss modulus at zero shear rate is not as deep as predicted by our model. Indeed one should expect a series of relaxation times instead of a single time due to the many particle interactions present in the material that cause excitation of many internal modes.

The results of this work can be compared with those obtained recently by van der Vorst et al. [5] for the steady shear viscosity of these dispersions. Although the model used in that study is completely different from the present one, they both result in values for the microrheological parameters $\phi_{\mathrm{cr}}$ and $\eta_{\mathrm{ff}}$. Therefore these values are also listed in Table II together with the parameter $R_{\mathrm{cr}}$. Both models show more or less the same dependence of the parameters upon the volume fraction and the rate of shear, as can be seen in Fig. 7. In the upper two panels of this figure the shear melting process is shown, while in the lower two not only the shear 
thinning behavior of the suspension but also that of the disordered fluid phase is shown. Although the models in both cases are rather crude, they capture the main features of the behavior of ordering latex suspensions in a steady shear flow.

It is possible to improve the model, but before that the next aspects have to be studied. First in the analysis of the experiment isotropy of the sample itself is assumed, but this is doubtful in the case of long range ordering in the sample. Second the variation of the steady shear rate over the gap due to the strong shear thinning behavior of the suspension gives rise to a systematic error in the measured $G^{*}$, which can be as large as a factor 2. However, the measurements do not change qualitatively when corrected. Considering this our model describes the measured linear viscoelastic behavior adequately. It corroborates the experimental results even quantitatively rather well, indicating that our hypothesis is useful in describing processes such as the one studied here. In order to obtain more quantitative results both the experimental method and the modeling will be studied in more detail in the future.

\section{APPENDIX: FORMAL CALCULATION OF $G^{*}$}

To calculate $G^{*}$ of the dispersion the result of Oosterbroek and Mellema [10] can be used directly. Since there is no interface present between aggregate and fluid all the interface parameters are set equal to zero and because $H \rightarrow \infty$ their expression for the complex viscosity [Eq. (A1.2)] reduces to

$$
\frac{\eta^{*}-\eta}{\eta}=\phi \frac{\gamma_{0}}{\gamma_{0}^{\prime}}
$$

where $\quad \gamma_{0}=C_{10} R^{10}+C_{3} R^{3} \quad$ and $\quad \gamma_{0}^{\prime}=C_{10}^{\prime} R^{10}+C_{7}^{\prime} R^{7}$ $+C_{5}^{\prime} R^{5}+C_{3}^{\prime} R^{3}+C_{0}^{\prime}$. In the present case $\eta=\eta_{\mathrm{fl}}^{*}$ and $\phi=\phi_{\mathrm{cr}}$. The coefficients $C_{n}$ reduce to

$$
\begin{gathered}
C_{3}=-190(E-1)^{2}, \\
C_{10}=+10(19 E+16)(E-1), \\
C_{0}^{\prime}=+76(E-1)^{2}, \\
C_{3}^{\prime}=-25(19 E+18)(E-1), \\
C_{5}^{\prime}=+42(19 E+16)(E-1), \\
C_{7}^{\prime}=-25(19 E+16)(E-1), \\
C_{10}^{\prime}=+2(19 E+16)(2 E+3) .
\end{gathered}
$$

Remembering $\phi_{\mathrm{cr}}=\left(f r_{a} / r_{c}\right)^{3}=f^{3} R^{-3}$ one obtains

$$
\begin{aligned}
G^{*} & =i \omega \eta^{*} \\
& =i \omega \eta_{\mathrm{fl}}^{*}\left(1+\frac{f^{3}\left(C_{10} R^{7}+C_{3}\right)}{C_{10}^{\prime} R^{10}+C_{7}^{\prime} R^{7}+C_{5}^{\prime} R^{5}+C_{3}^{\prime} R^{3}+C_{0}^{\prime}}\right),
\end{aligned}
$$

which is, after some manipulation, equivalent with Eq. (3).
[1] B. van der Vorst, D. van den Ende, and J. Mellema, J. Rheol. 39, 1183 (1995).

[2] L. B. Chen and C. F. Zukoski, J. Chem. Soc. Faraday Trans. 86, 2629 (1990).

[3] R. Buscall, Colloids Surf., A 83, 33 (1994).

[4] D. Quemada, Europhys. Lett. 25, 149 (1994).

[5] B. van der Vorst, D. van den Ende, N. J. J. Aelmans, and J. Mellema, Phys. Rev. E 56, 3119 (1997).

[6] A. Imhof, A. van Blaaderen, and J. K. G. Dhont, Langmuir 10, 3477 (1994).

[7] J. Zeegers, D. van den Ende, C. Blom, E. G. Altena, G. J. Beukema, and J. Mellema, Rheol. Acta 34, 606 (1995).

[8] R. I. Tanner and G. Williams, Rheol. Acta 10, 528 (1971).
[9] J. G. Oldroyd, Proc. R. Soc. London, Ser. A 218, 122 (1953).

[10] M. Oosterbroek and J. Mellema, J. Colloid Interface Sci. 84, 14 (1981).

[11] D. Graebling and R. Muller, J. Rheol. 34, 193 (1990).

[12] D. G. Thomas, J. Colloid Interface Sci. 20, 267 (1965).

[13] R. Simha, J. Appl. Phys. 23, 1020 (1952).

[14] J. W. Goodwin, J. Hearn, C. C. Ho, and R. H. Ottewill, Br. Polym J. 5, 347 (1973).

[15] J. Hearn, M. C. Wilkinson, and A. R. Goodall, Adv. Colloid Interface Sci. 14, 173 (1981).

[16] J. W. Goodwin and R. W. Hughes, J. Chem. Phys. 95, 6124 (1991). 